\title{
DETERMINAÇÃO DE COMPOSTOS BIOATIVOS EM ÓLEOS DE CHIA (SALVIA HISPÂNICA $\quad$ L.) E LINHAÇA (LINUM USITATISSIMUM, L.)
}

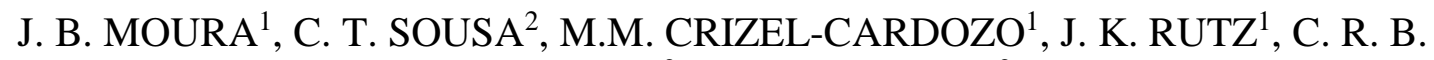 \\ MENDONÇA ${ }^{2}$, R. C. ZAMBIAZI². \\ ${ }^{1}$ Universidade Federal de Pelotas, Departamento de Ciência e Tecnologia Agroindustrial \\ ${ }^{2}$ Universidade Federal de Pelotas, Centro de Ciências Químicas, Farmacêuticas e de Alimentos \\ E-mail para contato: janice.moura@hotmail.com
}

RESUMO - O consumo de óleos vegetais vem substituindo o consumo de gorduras animais, por apresentarem propriedades benéficas à saúde provenientes de seus constituintes, como ácidos graxos, ômegas $(\omega)$, antioxidantes, tocoferóis dentre outros compostos. Devido a isso houve um aumento em pesquisas com espécies vegetais promissoras pouco exploradas como fonte de óleos alimentares. No presente estudo objetivou-se a determinação do teor de compostos fenólicos totais e tocoferóis de óleos vegetais prensados a frio de sementes de chia e linhaça comercializados na cidade de Pelotas. O conteúdo de compostos fenólicos totais encontrado nos óleos foi de 16,254 e $12,672 \mathrm{mg} / \mathrm{kg}^{-1}$ de ácido gálico para chia e linhaça respectivamente, o teor de tocoferóis obtido nos óleos de chia e linhaça foram respectivamente 1,126 e 0,963 ( $\delta$ tocoferol), 19,667 e 28,667 ( $\beta+\gamma$ tocoferol) e 11,480 ( $\alpha$ tocoferol), para o óleo de linhaça não foi observado quantidades detectáveis de $\alpha$ tocoferol.

\section{INTRODUÇÃO}

Hoje em dia, a tendência dos consumidores na escolha de alimentos esta associada à saúde e bem-estar, situação que pode ser claramente observada no mercado com o fornecimento de produtos distinguidos pelo seu conteúdo em ômega $(\omega)$, antioxidantes, fibras e outros componentes que os consumidores estão aprendendo a reconhecer como uma contribuição saudável (MARTINEZ et al., 2012).

Constituindo-se em um dos alimentos de maior contribuição para a ingestão de vitamina $\mathrm{E}$ para a população devido a suas altas concentrações de tocoferóis e tocotrienóis, os óleos vegetais vêm substituindo gradualmente o consumo de gorduras animais (NUNES, 2013; GUINAZ et al., 2009). Mas apesar da vasta gama de fontes de óleos vegetais, o consumo mundial é dominado por óleos de palma, soja, canola e girassol. Em função disso tem havido um aumento no desenvolvimento de espécies vegetais promissoras pouco exploradas como fonte de óleos alimentares (BARBOSA, 2011; IXTAINA et al., 2011). 


\section{9 a 22 de outubro de 2014 \\ Florianópolis/SC}

Com elevado potencial oleícola, as sementes de chia (Salvia hispânica L.) e linhaça (Linum usitatissimum , L.), representam rica fonte de ácidos graxos essenciais: ácido linolênico ( $\omega$-3) e ácido linoleico ( $\omega-6)$, além de antioxidantes naturais, tais como os compostos fenólicos exercendo diversos benefícios à saúde, tornando estas sementes alvo de pesquisas (GALVÃO et al., 2008; KASOTE; BADHE; HEGDE, 2013; URIBE et al., 2011; TOMBINI, 2013).

O objetivo do presente estudo foi determinar o teor de compostos fenólicos totais e tocoferóis de óleos vegetais comerciais de chia e linhaça.

\section{MATERIAIS E MÉTODOS}

As amostras de óleos vegetais foram adquiridas no comércio local de Pelotas/RS e levadas até o laboratório de cromatografia do departamento de ciência e tecnologia agroindustrial da Universidade Federal de Pelotas. Os óleos de chia e linhaça foram extraídos por prensagem a frio, sem sofrer processo de refino, segundo informação do fornecedor.

\subsection{Determinação de compostos fenólicos}

O conteúdo de compostos fenólicos totais dos óleos foi determinado segundo método adaptado de Gambocorta et al. (2010). Foi pesado 5g de cada uma das amostras e acrescentado 2 $\mathrm{ml}$ de metanol / água (70:30, v / v) e $2 \mathrm{~mL}$ de hexano, após foi misturado em vortex durante 1 min. e 20min. em shaker, a fase hidroalcoólica contendo os compostos fenólicos foi separada da fase oleosa por centrifugação $\left(7000 \mathrm{~g}, 4{ }^{\circ} \mathrm{C}, 10 \mathrm{~min}\right.$ ), a fase hidroalcoólica, foi submetida a centrifugação ( $7000 \mathrm{~g}, 4^{\circ} \mathrm{C}, 7 \mathrm{~min}$.) e finalmente recuperada.

Foi pipetado $500 \mu \mathrm{L}$ do extrato fenólico e acrescentado $500 \mu \mathrm{L}$ do reagente Folin-Ciocalteau $(0,2 \mathrm{~N})$, deixado em repouso por $4 \mathrm{~min}$. e adicionado $800 \mu \mathrm{L}$ de carbonato de sódio $\left(\mathrm{Na}_{2} \mathrm{CO}_{3}\right.$ a $5 \%$ ). A mistura foi então aquecida em banho de $40^{\circ} \mathrm{C}$ durante 20 minutos e o teor de fenóis totais foi determinado colorimetricamente a $750 \mathrm{~nm}$. A curva padrão foi preparada utilizando-se soluções diluídas de ácido gálico em metanol: água (70: 30, v / v). O conteúdo fenólico total foi expresso como equivalentes de ácido gálico (mg / 100g).

\subsection{Determinação de tocoferóis}

Para a determinação de tocoferóis as amostras de óleos foram diluídas com isopropanol até completar o volume no balão volumétrico de $25 \mathrm{~mL}$. Centrifugou-se por $10 \mathrm{~min}$ a $14.000 \mathrm{rpm}$ em microcentrífuga NT800 Nova Técnica. A análise dos tocoferóis foi realizada segundo metodologia descrita por Pestana et al. (2008).

Foi utilizado um sistema de cromatografia líquida de alta eficiência-HPLC (SHIMADZU) constituído por módulo de mistura dos solventes LC-10ATVP, desgaseificador FCV-10ALVP, bomba reodine DGU-14A, sistema de controle SCL-10AVP, forno da coluna CTO-10ASVP, e amostrador automático SIL-10AF. Utilizou-se uma coluna de separação analítica de fase reversa, Shim-Pak CLC-ODS $(3,9 \mathrm{~cm} \times 150 \mathrm{~mm} \times 4 \mu \mathrm{m})$, tendo como fase estacionária grupamentos octadesil. Utilizou-se o detector de fluorescência RF-10AXL, para a identificação dos tocoferóis. 
Os dados foram adquiridos e processados com o uso do software Class-VP.

\section{RESULTADOS E DISCUSSÕES}

\subsection{Determinação de compostos fenólicos}

O conteúdo de fenóis totais dos óleos de linhaça e chia prensados a frio é apresentado a seguir na Tabela 1.

Tabela 1- Teor de compostos fenólicos nos óleos de chia e linhaça.

\begin{tabular}{c|c}
\hline Óleos vegetais & Compostos fenólicos (mg/100g de ácido gálico) \\
\hline Chia & $1,625 \pm 1,56$ \\
Linhaça & $1,267 \pm 0,39$ \\
\hline
\end{tabular}

É possível observar que o óleo de chia apresenta um conteúdo mais elevado de compostos fenólicos em comparação ao óleo de linhaça.

Em estudos semelhantes com óleos de linhaça Borges et al. (2010) e Siger et al. (2008) obtiveram valores muito próximos ao resultado obtido neste estudo, sendo 1,2 e 1,14 mg/100g respectivamente o conteúdo total de compostos fenólicos.

Reyes-Caudillo et al. (2008) ao analisarem o conteúdo de compostos fenólicos totais em sementes de chia provenientes do estado de Jalisco no México obtiveram um total de 0,921mg/100g. Jiménez et al. (2010) ao avaliar o conteúdo de compostos fenólicos em óleo de chia, encontrou 5,92 equivalentes de ácido gálico (GAE).

\subsection{Determinação de tocoferóis}

$\mathrm{Na}$ separação cromatográfica observou-se três picos distintos (Figura 1), correspondentes aos componentes dos tocoferóis. Os tocoferóis foram identificados por comparação dos tempos de retenção com os padrões autênticos, identificou-se o $\delta$-, $\beta+\gamma$ - e $\alpha$-tocoferol, correspondentes aos picos 1, 2 e 3 respectivamente. No entanto, nas condições realizadas neste estudo o $\beta$ tocoferol não se separou de seu isômero, o $\gamma$-tocoferol, portanto, o pico 2 corresponde ao conteúdo do $\beta$ - e $\gamma$ - tocoferol. 


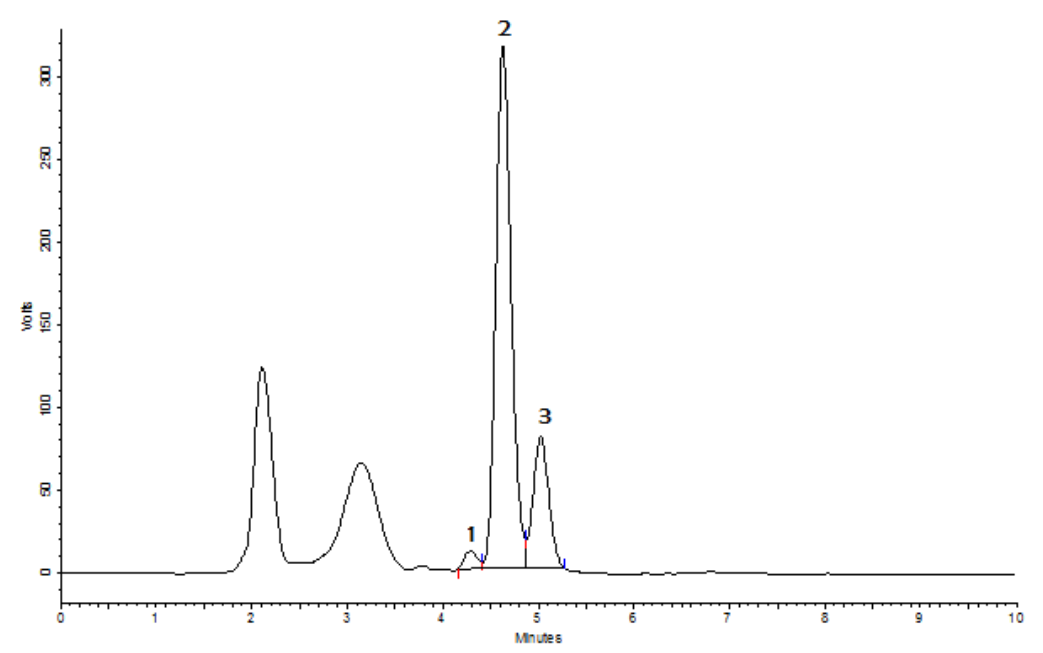

Figura 1: Cromatograma obtido por HPLC dos tocoferóis de óleo de chia. Identificação dos picos: (1) $\delta$-tocoferol; (2) $\beta+\gamma$-tocoferol e (3) $\alpha$-tocoferol.

Observa-se (Tabela 2) que o $(\gamma+\beta)$ - tocoferóis foram os compostos majoritários presentes tanto no óleo de chia quanto no óleo de linhaça, já o $\alpha$ tocoferol pode ser observado apenas no óleo chia.

Tabela 2- Teores de $\delta, \beta+\gamma$ e $\alpha$ tocoferóis nos óleos de chia e linhaça analisados.

\begin{tabular}{c|c|c|c}
\hline \multirow{2}{*}{ Óleos } & \multicolumn{3}{|c}{ Tocoferol (mg/100g) } \\
\cline { 2 - 4 } & $\delta$ tocoferol & $\beta+\gamma$ tocoferol & $\alpha$ tocoferol \\
\hline Chia & $1,126 \pm 0,05$ & $19,667 \pm 0,22$ & $11,480 \pm 0,17$ \\
Linhaça & $0,963 \pm 0,03$ & $28,667 \pm 0,67$ & $*$ ND \\
\hline
\end{tabular}

*ND Não detectado.

Os valores totais de tocoferóis entre os óleos variaram cerca de $2,6 \mathrm{mg} / 100 \mathrm{~g}$, sendo o óleo de chia a amostra com o maior valor total com $32,267 \mathrm{mg}$.

Em estudo semelhante Schwartz et al. (2008) avaliou o conteúdo de tocoferóis de diferentes óleos comerciais, e obteve o valor $52 \mathrm{mg} / 100 \mathrm{~g}$ de $\gamma$ tocoferol, $0,95 \mathrm{mg} / 100 \mathrm{~g}$ de $\delta$ tocoferol e $1,2 \mathrm{mg} / 100 \mathrm{~g}$ de $\alpha$ tocoferol para o óleo de linhaça.

Martínez et al. (2012) ao extrair o óleo de chia com temperaturas variando de 30 a $70^{\circ} \mathrm{C}$, analisou o teor total de tocoferóis presentes, obtendo um valor de $50,2 \mathrm{mg} / 100 \mathrm{~g}$.

\section{CONCLUSÕES}




\section{9 a 22 de outubro de 2014 \\ Florianópolis/SC}

O óleo de linhaça apresentou teor de fenóis semelhante a outros trabalhos, porém o óleo de chia apresentou valores inferiores. A análise de tocoferóis apresentou valores inferiores aos reportados por outros autores, no entanto vários fatores devem ser levados em consideração como metodologia aplicada, método de extração, equipamento utilizado dentre outros fatores. Entre os óleos avaliados, o óleo de chia apresentou um conteúdo de compostos bioativos levemente superior ao óleo de linhaça.

\section{REFERÊNCIAS}

BARBOSA, M. Z. Óleos Vegetais para Alimentos ou para Biodiesel? Análises e Indicadores do Agronegócio, São Paulo, v.6, n.6, 2011.

BORGES, L. R.; HELBIG, E.; BUENO, F. M.; ROSA, C. G.; PEREIRA, M. R.; ZAMBIAZI, R. C. Avaliação da qualidade e conteúdo de fenóis do óleo de linhaça prensado a frio. In: XII ENPOS E XIX CONGRESSO DE INICIAÇÃO CIENTÍFICA, 2010, Pelotas. Anais do XII Enpos e XIX Congresso de iniciação científica, Pelotas, 2010.

GAlVÃO, E. L.; SILVA, D. C. F.; SILVA, J. O.; MOREIRA, A. V. B.; SOUSA, E. M. B. D. Avaliação do potencial antioxidante e extração subcrítica do óleo de linhaça. Ciência $e$ Tecnologia de Alimentos, Campinas, p. 551-557, 2008.

GAMBOCORTA, G.; FACCIA, M.; PREVITALI, M. A.; PATI, S.; LA NOTTE, E.; BAIANO, A. Effects of olive maturation and stoning on quality indices and antioxidant content of extra virgin oils (cv. Coratina) during storage. Journal of food science, v.75, n.3, p.229-235, 2010.

GUINAZ, M.; MILAGRES, R. C. R. M.; PINHEIRO-SANT'ANA, H. M.; CHAVES, J. B. P. Tocoferóis e tocotrienóis em óleos vegetais e ovos. Química Nova, São Paulo, v.32, n.8, 2009.

IXTAINA, V. Y.; MARTÍNEZ, M. L.; SPOTORNO, V.; MATEO, C. M.; MAESTRI, D. M.; DIEHL, B. W.; NOLASCO, S. M.; TOMÁS, M. C. Characterization of chia seed oils obtained by pressing and solvent extraction. Journal of Food Composition and Analysis, v.24, p.166-174, 2011.

JIMÉNEZ, F. E. G.; BELTRÁN-OROZCO, M. C.; MARTÍNEZ, V. M. G. The antioxidant capacity and phenolic content of chía's (Salvia hispánica L.) integral seed and oil. Journal of Biotechnology, México, v.150, p. 315, 2010.

KASOTE, D. M.; BADHE, Y. S.; HEGDE, M. V. Effect of mechanical press oil extraction processing on quality of linseed oil. Industrial Crops and Products, India, v.42, p.10-13, 2013.

MARTINEZ, M. L.; MARIN, M. A.; FALLER, C. M. S.; REVOL, J.; PENCI, M. C.; RIBOTTA, P. D. Chia (Salvia hispanica L.) oil extraction: Study of processing parameters. LWT - Food Science and Technology, Argentina, v.47, p.78-82, 2012.

NUNES, S. P. Produção e consumo de óleos vegetais no Brasil. 2 out. 2013. Acessado em 2 out.

2013. Online. Disponível em: http://www.deser.org.br/documentos/doc/Produ\%E7\%E3o\%20e\%20consumo\%20de\%20\%F3leo s\%20vegetais.pdf 
PESTANA, V.R.; ZAMBIAZI, R.C.; MENDONÇA, C.R. BRUSCATTO, M.H.; LERMAGARCIA, M.J; RAMIS-RAMOS, G. Quality Changes and Tocopherols and $\gamma$-Orizanol Concentrations in Rice Bran Oil During the Refining Process. J. Am. Oil Chem. Soc, v.85, p. 1031019, 2008.

REYES-CAUDILLO, E.; TECANTE, A.; VALDIVIA-LÓPEZ,M.A. Dietary fibre content and antioxidant activity of phenolic compounds present in Mexican chia (Salvia hispanica L.) seeds. Food Chemistry, v.107, p.656-663, 2008.

SCHWARTZ, H.; OLLILAINEN, V.; PIIRONEN, V.; LAMPI, A. M. Tocopherol, tocotrienol and plant sterol contents of vegetable oils and industrial fats. Journal of Food Composition and Analysis, Viena, v.21, p. 152-161, 2008.

SIGER, A.; NOGALA-KALUCKA, M.; LAMPART-SZCZAPA, E. The content and antioxidant activity of phenolic compounds in cold-pressed plant oils. Journal of Food Lipids. v. 15, p. 137149, 2008.

TOMBINI, J. Aproveitamento tecnológico da semente de chia (Salvia Hispanica L.) na formulação de barra alimentícia. 2013. 36f. Monografia (Bacharel em Química) - Universidade Tecnológica Federal do Paraná, Pato Branco.

URIBE, J. A. R.; PEREZ, J. I. N.; KAUIL, H. C.; RUBIO, G. R.; ALCOCER, C. G. Extraction of oil from chia seeds with supercritical $\mathrm{CO}_{2}$. The Journal of Supercritical Fluids, México, v.56, p.174-178, 2011. 